\title{
Continuous Legged Locomotion Planning
}

\author{
Nicolas Perrin, Christian Ott, Johannes Englsberger, Olivier Stasse, Florent Lamiraux and Darwin G. Caldwell
}

\begin{abstract}
While only continuous motions are possible, the way contacts appear and disappear confers to legged locomotion a characteristic discontinuous nature that is traditionally shared by the algorithms used for legged locomotion planning. In this paper we show that this discontinuous nature can disappear if the notion of collision is well redefined, and we efficiently solve two different practical problems of legged locomotion planning with algorithms based on an approach that establishes a bridge between discrete and continuous planning. The first problem consists of reactive footstep planning with a biped robot, and the second one of non-gaited locomotion planning with a hexapod.
\end{abstract}

\section{INTRODUCTION}

Technically, the configuration space of a legged robot can naturally be seen as the union of an infinity of submanifolds, and thus can be refered to as "stratified". Indeed, when a set of (driftless) contacts between the robot and the ground is fixed, the possible motions belong to a submanifold entirely defined by the contacts. When a contact with the ground is suddenly made or released, the system immediately moves from a submanifold to another, and the equations of motion change in a discrete manner (some constraints due to the contact modeling are abruptly created or removed). As pointed out in [10], this discontinuous nature is one of the most important characteristics of legged robots (and other "stratified systems" whose configuration spaces are similar). This explains why the majority of simplified models that are used to solve locomotion planning problems in a computationally efficient way also have a discontinuous nature. Completely continuous models have been used in previous works (see [29], [16], [8]), but they always fail to capture some important features of the legged robots, such as their ability to step over obstacles.

In [10], small-time local controllability is obtained for some stratified systems thanks to an extension of the ChowRashevskii theorem ([4], [27]). As a result, some classical motion planning algorithms based on controllability, such as [20], can be applied to locomotion planning for legged robots (see [11] and [13]), but it essentially consists in converting initial sliding motions into feasible walking motions. Again, this means that the robot is not able to step over obstacles.

N. Perrin is with (1) Sorbonne Universités UPMC Univ. Paris 06, UMR 7222, ISIR, Paris, France, (2) CNRS, UMR 7222, ISIR, F-75005, Paris, France e-mail: perrineisir.upmc.fr.

C. Ott and J. Englsberger are with Institute of Robotics and Mechatronics, German Aerospace Center (DLR), Wessling, Germany e-mail: \{christian.ott, johannes.englsberger\}edlr.de.

O. Stasse and F. Lamiraux are with CNRS/LAAS, Université de Toulouse UPS, INSA, INP, ISAE, Toulouse, France e-mail: \{ostasse, florent\}alaas. fr.

D. G. Caldwell is with Istituto Italiano di Tecnologia, Via Morego 30, 16163 Genova e-mail: darwin. caldwelleit.it.
Continuous models are, however, interesting because they make possible the use of conventional, efficient motion planning algorithms that suppose smooth configuration spaces (e.g. PRM [17], RRT [21]). Currently, algorithms that take into account the discontinuous nature of stepping motions usually choose the footsteps before computing the motions. Depending on the context and on the robot, a heuristic is defined and used to search for finite sequences of footsteps that solve the motion requirements. Once a sequence of footsteps has been determined, the next phase is to find a feasible continuous motion of the robot that follows this sequence. This approach means that the discrete and continuous aspects of the problem are considered separately. This method has been used extensively for humanoid robot navigation planning ([19], [2], [5], [6], [12]), for locomotion planning for a hexapod [14], or for more general multi-contact planning problems [3]. To search for finite sequences of footsteps, since conventional motion planning algorithms cannot be applied, graph search algorithms such as $\mathrm{A}^{*}$ are used instead.

In this paper, which is a short version of [24], we present an approach that can take into account the discrete aspect of walking while simultaneously permitting motion planning in a smooth configuration space. This method is based on an abstract equivalence derived from previous work [23] that can be instantiated in different contexts to transform discrete planning problems into continuous ones. In our applications of legged locomotion planning, the equivalence is between the discrete problem of planning sequences of footprints in the $2 \mathrm{D}$ space and the planning of continuous translations and rotations for a particular 2D shape. The geometrical properties of the shape highly depend on the geometry of the robot (more details are given in the next section). For example, for biped walking we choose a shape made of two portions of disk, and for a hexapod the disjoint union of 6 disks. Classical algorithms such as RRT or PRM can be used to plan the continuous motion of the shape, and a greedy algorithm then transforms it into a sequence of steps. Fig. 1 gives an overview of the global approach.

Our work shares similarities with [1] where a connection is established between the free space of a spider robot and the free space of a half-disk robot moving by translation and rotation amidst obstacles, but the goal in their work was to solve exactly the motion planning problem on a specific and relatively abstract example. Our approach is different in many aspects: first, we base our results on a very generic equivalence that can be applied to various kinds of legged robots (i.e. with different number of limbs or different leg configurations). Then, we focus on the principle of changing the notion of collision, which is a new way to tackle the problem. Finally, instead of looking for exact solutions, we aim at efficiently using sampling-based motion planning algorithms in practical 
STEP 0: Foundation of the method: a generic equivalence between discrete and continuous motion planning problems (see [22] and [23]).

STEP 1: the context is defined by the robot and its stepping capabilities.

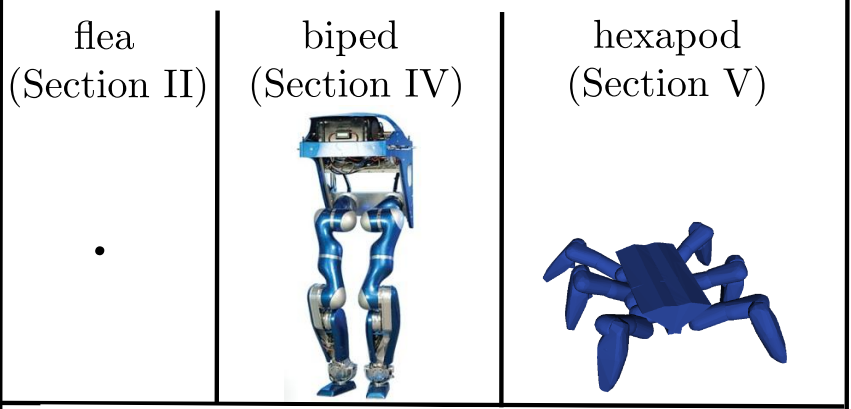

STEP 2: a simple and appropriate geometrical "shape" slightly restricts the steps the robot can make and allows for an instanciation of the equivalence.
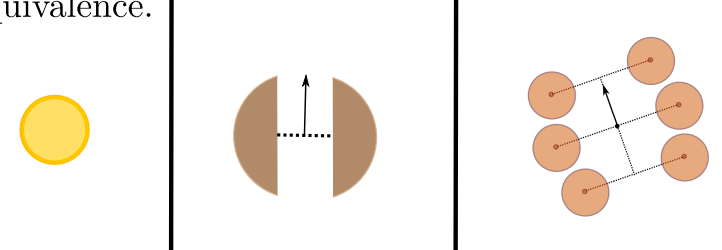

STEP 3: definition of a new notion of collision and use of classical algorithms such as RRT or PRM to find continuous "weakly collision-free" paths.

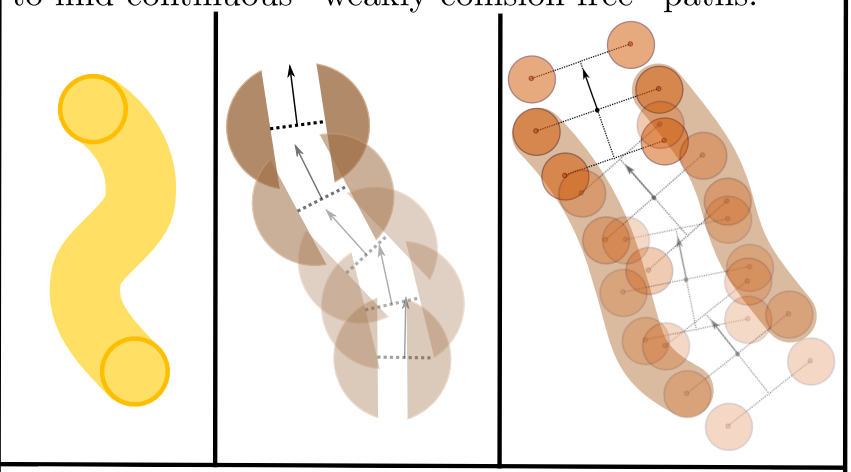

STEP 4: a greedy algorithm converts the continuous paths into discrete sequences of steps.

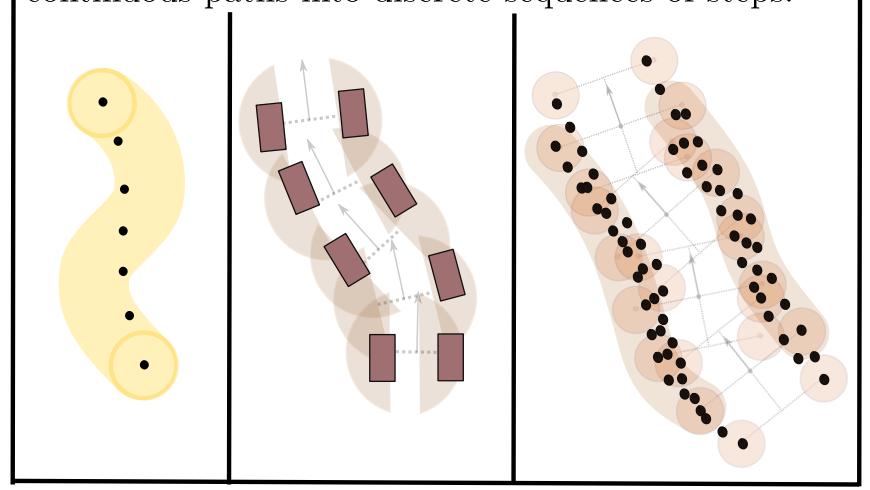

Fig. 1. Overview of the method. scenarios.

In Section II, we define the problem of "flea motion planning", demonstrate a theorem of equivalence between discrete and continous motion planning for the flea, and present an efficient algorithm, based on the concept of weak collisionfreeness, that illustrates our approach. Complexity questions related to the use of this concept are discussed in Section III. In Section IV we briefly explain how our algorithm for flea motion planning can be seen as an instance of a more general result presented in [23] and [24], which can be applied to transform various discrete motion planning problems into continuous ones. In Section V, we present an application of this result to vision-based reactive footstep planning with the DLR-Biped robot, and in Section VI, we show that the same approach can be quite easily adapted to locomotion planning for a hexapod robot. This possibility of generalization to multilegged robots is an important component of our contribution. Indeed, while $\mathrm{A}^{*}$-based approaches work relatively well with biped robots, their efficiency decreases rapidly when the number of legs increases, because of the high sensitivity to the branching factor. On the contrary, our approach scales well and, in the example considered, results in an efficient algorithm that quickly produces hexapod motions on complex terrains without needing any prior knowledge on hexapod gaits. Section VII concludes the paper.

\section{THE PROBLEM OF FLEA MOTION PLANNING, AND AN EFFICIENT ALGORITHM TO SOLVE IT}

To illustrate the principle of our approach, we consider a toy problem that we call "flea motion planning" and which best exemplifies the key ingredient of our method: although the problem seems intrinsically discontinuous, changing the notion of collision transforms it into an entirely continuous one, which then allows the use of classical continuous motion planning algorithms to solve it efficiently.

The flea is represented by a point in a $2 \mathrm{D}$ environment; $\mathcal{C}=\mathbb{R}^{2}$ is the configuration space. For the flea, we use the terms position and configuration as synonyms. There are obstacles in this 2D environment such that the free space $\mathcal{F}$ is an open set. The flea can make jumps (steps) in any direction and of any length strictly less than $l_{\max }>0$. The goal is to find a sequence of jumps from one configuration $\left(x_{A}, y_{A}\right) \in \mathcal{F}$ to a second configuration $\left(x_{B}, y_{B}\right) \in \mathcal{F}$ such that every intermediate "landing point" of the flea is in $\mathcal{F}$. The discontinuous nature of the jumps in this flea motion planning problem is comparable to the discontinuous nature of the problem of finding sequences of suitable footsteps for a walking machine.

We start by proving an equivalence between the discontinuous jumping motions of the flea, and the continuous motions of an open disk, but with a new notion of collisionfreeness. So, let us assume that a sequence of jumps (steps) has been found, and that it corresponds to the sequence of configurations $p_{1}=\left(x_{1}, y_{1}\right), p_{2}, p_{3}, \ldots, p_{n}=\left(x_{n}, y_{n}\right)$, with $\left(x_{1}, y_{1}\right)=\left(x_{A}, y_{A}\right)$ and $\left(x_{n}, y_{n}\right)=\left(x_{B}, y_{B}\right)$. We consider the continuous motion of an open disk of diameter $l_{\text {max }}$ such that the trajectory $(x(t), y(t))_{t \in[0,1]}$ of its center 


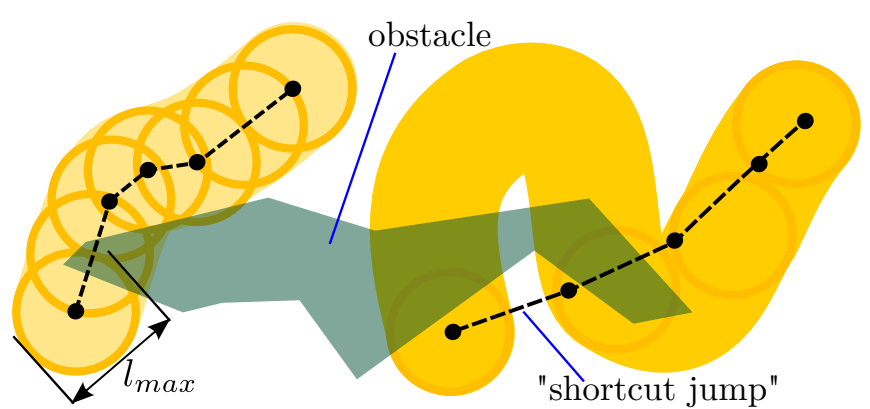

Fig. 2. The "flea motion planning problem". On the left: from a collision-free sequence of flea jumps to a continuous "weakly collision-free" path for the disk. On the right: converting a continuous weakly collision-free path of the disk into a sequence of flea jumps, using a greedy algorithm.

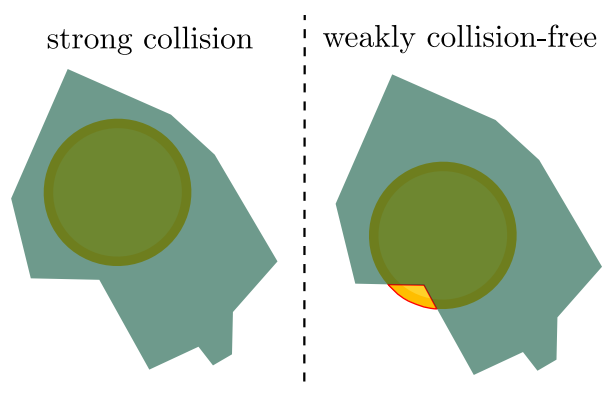

Fig. 3. Weak collision-freeness.

follows a sequence of line segments between each $\left(x_{i}, y_{i}\right)$ and $\left(x_{i+1}, y_{i+1}\right)$ for $i=1,2, \ldots, n-1$, as depicted in Fig. 2 (on the left). An interesting property of this continuous disk motion is the following (it is a direct consequence of the upper bound $l_{\max }$ on the length of jumps):

Property 1: For all $t \in[0,1]$, the disk of center $(x(t), y(t))$ contains at least one of the flea configurations $p_{1}, p_{2}, \ldots, p_{n}$. This property suggests the definition of a new notion of collision-freeness:

Definition 1: Let us denote by $\mathcal{D}_{(x, y)}$ the open disk of center $(x, y)$ and diameter $l_{\max }$. We say that a disk $\mathcal{D}_{(x, y)}$ is collision-free if there exists at least one flea configuration (i.e. point) inside the disk which is collision-free. We call this new notion of collision-freeness the "weak collision-freeness", and say that the disk is "weakly collision-free". Conversely, if all the flea configurations (points) inside the disk are in collision (i.e. the disk does not intersect the free space), we say that the disk is in "strong collision". We say that a continuous path $\left(\mathcal{D}_{(x(t), y(t))}\right)_{t \in[0,1]}$ is weakly collision-free if for every $t \in[0,1], \mathcal{D}_{(x(t), y(t))}$ is weakly collision-free.

Fig. 3 illustrates this definition. A direct consequence of Property 1 is the following theorem:

Theorem 1: If there exists a finite sequence of collisionfree jumps from $\left(x_{A}, y_{A}\right)$ to $\left(x_{B}, y_{B}\right)$, then there also exists a weakly collision-free continuous path $\left(\mathcal{D}_{(x(t), y(t))}\right)_{t \in[0,1]}$ such that $(x(0), y(0))=\left(x_{A}, y_{A}\right)$ and $(x(1), y(1))=\left(x_{B}, y_{B}\right)$.

Proof: Let $\left(\mathcal{D}_{(x(t), y(t))}\right)_{t \in[0,1]}$ be a path such that the center of the disk follows the line segments between the consecutive collision-free landing points in a sequence of jumps from $\left(x_{A}, y_{A}\right)$ to $\left(x_{B}, y_{B}\right)$. As mentioned above, such a

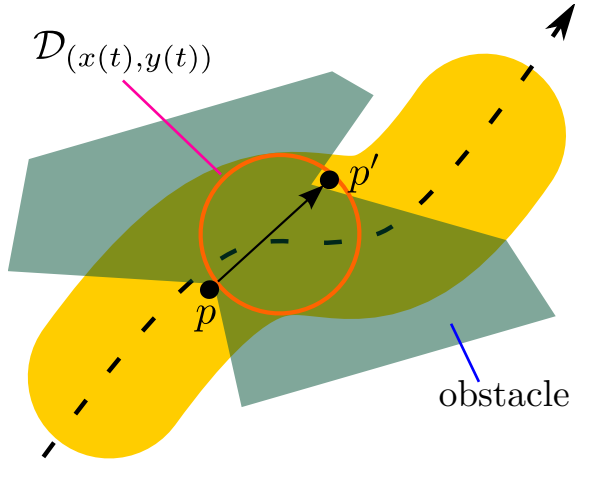

Fig. 4. Progression inside a weakly collision-free path. The current collisionfree position of the flea is $p$. A bit further along the path, we can choose a disk $\mathcal{D}_{(x(t), y(t))}$ such that $p$ is outside of it but arbitrarily close to its boundary. This disk is weakly collision-free, so its intersection with the free space is non-empty. If the disk is close enough to $p$, it is possible to find a position $p^{\prime}$ in this intersection such that the flea can directly jump from $p$ to $p^{\prime}\left(d_{2}\left(p, p^{\prime}\right)<l_{\max }\right)$. This is the reason why the flea can always move forward along weakly collision-free paths.

path verifies Property 1 , which means that for every $t \in[0,1]$, $\mathcal{D}_{(x(t), y(t))}$ contains at least one collision-free configuration and is therefore weakly collision-free. As a consequence the entire path is by definition weakly collision-free.

We prove that the converse of Theorem 1 is also true:

Theorem 2: If there exists a weakly collision-free continuous path $\left(\mathcal{D}_{(x(t), y(t))}\right)_{t \in[0,1]}$ from $\left(x_{A}, y_{A}\right)$ to $\left(x_{B}, y_{B}\right)$ (with $(x(0), y(0))=\left(x_{A}, y_{A}\right)$ and $\left.(x(1), y(1))=\left(x_{B}, y_{B}\right)\right)$, then there exists a finite sequence of collision-free jumps from $\left(x_{A}, y_{A}\right)$ to $\left(x_{B}, y_{B}\right)$.

Proof: Intuitively, the reason for this theorem to hold is that since the path is weakly collision-free, the free space intersects every disk along that path. Since the flea can make jumps as large as the diameter of these disks $\left(l_{\max }\right)$, it can always move forward while staying inside the free space. Fig. 4 illustrates this property.

Let us denote by $d_{2}$ the Euclidean distance in $\mathbb{R}^{2}$. For a point $p \in \mathcal{C}$ we denote by $d_{\text {obs }}(p)=\inf \left\{d_{2}(p, o) \mid o \in \mathcal{C} \backslash \mathcal{F}\right\}$ its distance to the obstacles. For a disk $\mathcal{D}_{(x, y)}$ we define:

$$
\delta_{o b s}\left(\mathcal{D}_{(x, y)}\right)=\sup \left\{d_{o b s}(p) \mid p \in \mathcal{D}_{(x, y)}\right\} .
$$

A disk $\mathcal{D}_{(x, y)}$ is weakly collision-free if and only if $\delta_{\text {obs }}\left(\mathcal{D}_{(x, y)}\right)>0$. Let us consider a weakly collision-free continuous path $\left(\mathcal{D}_{s(t)}\right)_{t \in[0,1]}$ from $\left(x_{A}, y_{A}\right)$ to $\left(x_{B}, y_{B}\right)$, with $s(t)=(x(t), y(t))$.

We define $d_{\text {inf }}=\frac{1}{2} \inf \left\{\delta_{o b s}\left(\mathcal{D}_{s(t)}\right) \mid t \in[0,1]\right\}$. By continuity of $t \mapsto \delta_{o b s}\left(\mathcal{D}_{s(t)}\right)$, we have $d_{\text {inf }}>0$. By uniform continuity of $t \mapsto s(t)$, there exists $0<\epsilon<1$ such that $\forall t \in[0,1-\epsilon]$, $\forall \epsilon^{\prime} \in[0, \epsilon], d_{2}\left(s(t), s\left(t+\epsilon^{\prime}\right)\right)<\min \left(d_{\mathrm{inf}}, l_{\max }\right)$.

Let us now consider $t \in[0,1-\epsilon]$ and a collision-free configuration $p$ of the flea in $\mathcal{D}_{s(t)}$. First, we know that there exists $p^{\prime} \in \mathcal{D}_{s(t)}$ such that $d_{o b s}\left(p^{\prime}\right)>d_{\text {inf }}$. Besides, since $\mathcal{D}_{s(t)}$ is of diameter $l_{\max }$, we have $d_{2}\left(p, p^{\prime}\right)<l_{\max }$, and thus the flea can jump from $p$ to $p^{\prime}$. Then, since we have $d_{2}\left(s(t), s\left(t+\frac{1}{M}\right)\right)<\min \left(d_{\text {inf }}, l_{\max }\right)$ for some $M \in \mathbb{N}_{>0}$ such that $\frac{1}{M}<\epsilon$, there exists $p^{\prime \prime} \in \mathcal{D}_{s\left(t+\frac{1}{M}\right)}$ such that $d_{2}\left(p^{\prime}, p^{\prime \prime}\right)<\min \left(d_{\mathrm{inf}}, l_{\max }\right)$. It follows that the flea can 
jump from $p^{\prime}$ to $p^{\prime \prime}$ and, since $d_{o b s}\left(p^{\prime}\right)>d_{\text {inf }}$, that $p^{\prime \prime}$ is collision-free. So we have proved that if $p$ is a collision-free configuration of the flea in $\mathcal{D}_{s(t)}$ with $t \in\left[0,1-\frac{1}{M}\right]$, it is always possible to reach a collision-free configuration in $\mathcal{D}_{s\left(t+\frac{1}{M}\right)}$ with at most 2 jumps. By induction, we deduce that a collision-free configuration $p_{\alpha} \in \mathcal{D}_{s(1)}$ can be reached after no more than $2 M$ jumps. We have $d_{2}\left(p_{\alpha},\left(x_{B}, y_{B}\right)\right)<l_{\max }$, thus the flea can jump directly from $p_{\alpha}$ to $\left(x_{B}, y_{B}\right)$. This concludes the proof, and an example of sequence of jumps obtained from a weakly collision-free continuous path of the disk can be seen on the right side of Fig. 2.

Together, Theorem 1 and Theorem 2 form an equivalence between the weakly collision-free paths of the disk and the collision-free sequences of jumps of the flea.

It turns out that this equivalence gives an efficient algorithm to solve the flea motion planning problem. Indeed, instead of looking for a discontinuous sequence of jumps, we can first look for a continuous path of the disk, and that can be done with any conventional motion planning algorithm, provided that we implement new collision checks using Definition 1. To convert a continuous path into a finite sequence of jumps, we can apply a greedy approach already used in previous work [25] that consists in repeatedly trying to jump from the current disk $\mathcal{D}_{s(t)}$ to a disk $\mathcal{D}_{s\left(t^{\prime}\right)}$ with $t^{\prime}$ as large as possible and obtained by dichotomy. This can result in "shortcut jumps", as shown in Fig. 2 (on the right).

Using such a transfer towards continuous motion planning has several benefits.

For the flea motion planning problems, other more direct approaches are also very efficient, and for example it would be easy to naively adapt the RRT algorithm to grow trees of discrete sequences of jumps. But for more complicated problems such as footstep planning, adapting RRT is not an easy task because it is not always clear how to extend a node towards a sample configuration. For this reason the current state-of-the-art method is to choose in advance a finite set of possible steps and then use $\mathrm{A}^{*}$-like algorithms to search for sequences of steps towards a goal ([15], [19], [2], [5], [6]). Although adjustments have been considered (see [7]), starting by manually selecting a finite number of possible steps is not very satisfying and leads to several problems such as limited stepping capabilites. The approach we present in Section V in the context of humanoid robot footstep planning is based on a similar equivalence as the one formed by Theorem 1 and Theorem 2, and it enables to deal with fully continuous stepping capabilities in an efficient and theoretically sound way.

For a hexapod, a large set of possible steps would be required in order to obtain decent stepping capabilities, and therefore methods based on $\mathrm{A}^{*}$-like algorithms are difficult to apply. This problem can be circumvented by using fixed gaits, but they are less efficient in complex environments. The method we propose in Section VI is again based on an equivalence that transforms the problem into a fully continuous one, and it enables to very quickly find sequences of steps even in quite complex environments.

\section{COMPlEXITY OF THE COLLISION CHECKS}

With 2D flea motion planning, checking whether a disk $\mathcal{D}_{(x, y)}$ is weakly collision-free or not can be done quite efficiently by shrinking the obstacles and computing a standard collision check for the center of the disk, $(x, y)$. However, as we will see in the following sections, in the general case the disk is replaced by a set of configurations $\mathcal{U}_{\chi}$ (depending on a vector of parameters $\chi$ ), which is weakly collision-free if there exists one configuration in it that is collision-free (in a standard way). This addition of an existential quantifier to the collision checks has obviously a computational cost, but using slightly conservative approximations allows efficient implementations.

Indeed, choosing an arbitrary threshold (e.g. 90\%), we can use a constant number of random collision checks to either find a collision-free configuration in the set $\mathcal{U}_{\chi}$, or state with high-confidence that more than $90 \%$ of randomly selected configurations in $\mathcal{U}_{\chi}$ are in collision. This results in an approximated check of weak collision-freeness that cannot lead to false positives.

Furthermore, as $\mathcal{U}_{\chi}$ is modified continuously, two sets $\mathcal{U}_{\chi\left(t_{1}\right)}$ and $\mathcal{U}_{\chi\left(t_{2}\right)}$ tend to have a large overlap if $\left|t_{2}-t_{1}\right|$ is small. So, a collision-free configuration inside $\mathcal{U}_{\chi\left(t_{1}\right)}$ can prove that $\mathcal{U}_{\chi(t)}$ is weakly collision-free for a whole range of values of $t$ close to $t_{1}$. As a result, a buffer of collision-free configurations can save a significant amount of computation time, allowing several evaluations to be done with few standard collision checks.

This works well if the sets $\mathcal{U}_{\chi}$ are often weakly collisionfree, but for sets in strong collision the same constant number of checks is always needed, and this number increases with the threshold chosen (90\% in the above example). In methods like RRT or PRM, the collision checks can be divided into two categories: milestone configurations, and collision checks along paths between milestones. To avoid spending too much computation time checking configurations in collision, we can use a different threshold for the two categories, for instance by choosing a threshold of $50 \%$ for milestone sets (thus decreasing the number of standard collision checks performed before declaring that a set is in strong collision), and using a much higher threshold in the paths between milestones, as the weak collision-freeness of the milestones increases the probability that parameters in between lead to sets that are weakly collision-free as well.

Using these approximations, the overall complexity of the approach (in terms of number of collision checks) becomes comparable to that of the continuous motion planning algorithm used. The second phase, that greedily computes the sequence of steps based on the continuous path, has a computation times that is in practice negligible compared to the planning phase.

\section{GeneralizATION OF THE EQUIVALENCE BETWEEN DISCRETE AND CONTINUOUS MOTION PLANNING}

The theorems and algorithms presented in Section II describe well the very core of our approach, but they are specific to flea motion planning. To adapt our method to various practical problems, we need a more general approach that can 


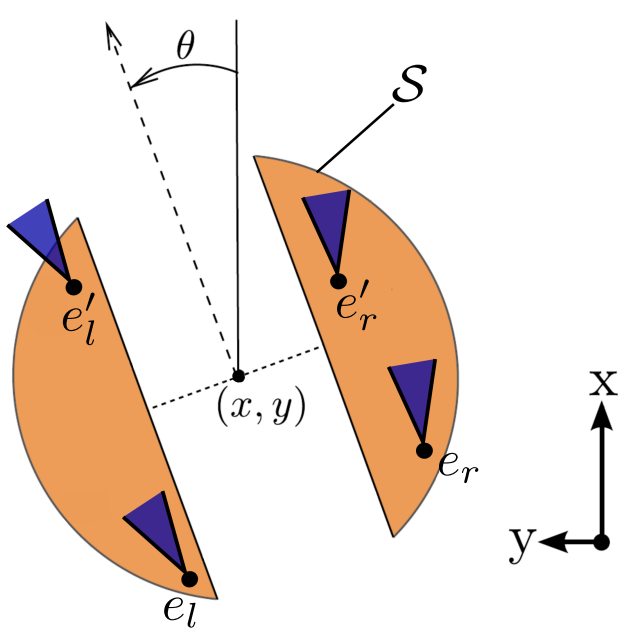

Fig. 5. The shape $\mathcal{S}$ on which we base our definition of the transition relation $R$ in the case of humanoid footstep planning.

transform various types of discrete motion planning problems into continuous ones.

Theorem 3.1 in [24] gives a list of abstract conditions that yield equivalences between discrete and continuous motion planning problems. This theorem is a generalization of the equivalence obtained with the flea motion planning problem. Without going into details, a discrete motion planning problem is characterized by a transition relation $R\left(p, p^{\prime}\right)$ which evaluates to true if and only if it is possible to discretely "jump" from configuration $p$ to configuration $p^{\prime}$. For such a discrete motion planning problem, we try to find a new space $\Omega$ and sets of configurations $\mathcal{U}_{\chi \in \Omega}$ (corresponding to the disks $\mathcal{D}_{(x, y)}$ in the case of the flea), as well as a new notion of weak collision-freeness for these sets, such that any valid, collisionfree sequence of jumps can be transformed into a continuous trajectory of weakly collision-free sets $\mathcal{U}_{\chi}$, and vice versa. The general theorem presented in [24] (and in another version in [23]) provides sufficient conditions for the existence of such transformations, but it does not explain how the space $\Omega$ and the sets $\mathcal{U}_{\chi}$, or even the notion of weak collision-freeness, should be defined. Some intuition can be found in the proof of the theorem, but there are no clear limitations nor obvious patterns in the structure of $\Omega$ and the sets $\mathcal{U}_{\chi}$. When trying to obtain a specific equivalence for a concrete problem, the most essential things to have in mind are probably that:

1) all the key ingredients of the approach can be found in the equivalence for the flea motion planning problem,

2) the relation $R$ and the sets $\mathcal{U}_{\chi}$ are strongly related,

3) a few restrictions on the transitions can give symmetries to $R$ that make the equivalence easier to get.

In the next two sections, we present examples of concrete applications of our approach.

\section{ApPliCATION to REACTIVE HUMANOID FOOTSTEP PLANNING}

To apply our method to bipedal walking, we first need to understand how the problem is related to flea motion planning.

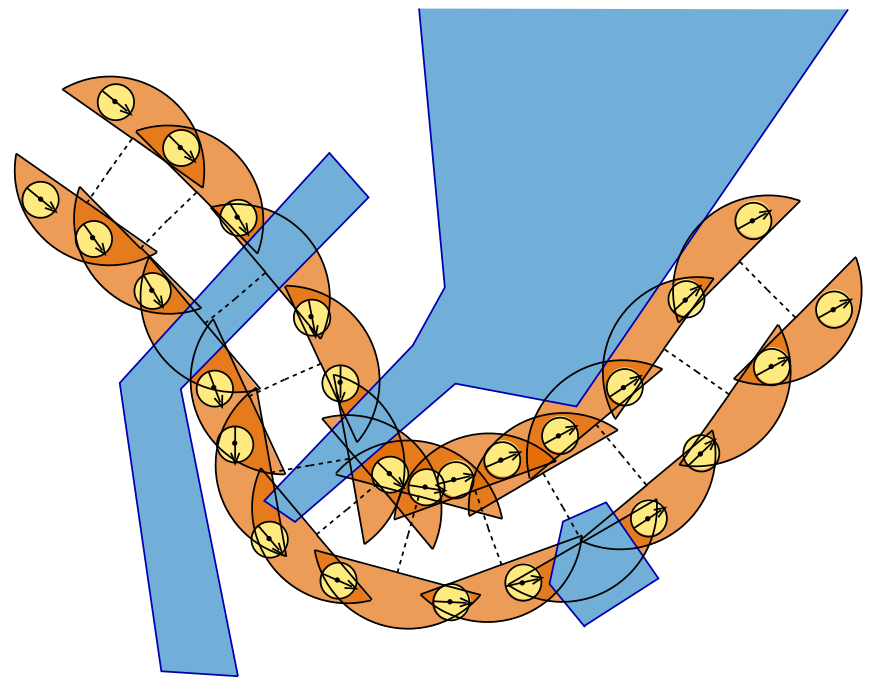

Fig. 6. Conversion from a weakly collision-free continuous path in $S E(2)$ to a valid finite sequence of steps.

The configurations of the flea are $2 \mathrm{D}$ points, whereas the intermediate postures of a biped robot can be represented by the position and orientation of each foot, i.e. two elements of $S E(2)$, the special Euclidean group of rigid-body motions in the plane. Instead of $\mathbb{R}^{2}, \mathcal{C}$ becomes $S E(2)^{2}$, with configurations of the robot feet (or stances) being written $\left(e_{l}, e_{r}\right)$, with $e_{l} \in S E(2)$ the position and orientation of the left foot, and $e_{r} \in S E(2)$ the position and orientation of the right foot. The relation $R\left(\left(e_{l}, e_{r}\right),\left(e_{l}^{\prime}, e_{r}^{\prime}\right)\right)$ must describe realistic stepping capabilities of the robot, and as pointed out in the previous section, it is convenient when $R$ is simple and has symmetries. Aiming at an equivalence similar to the one for the flea, and considering also the actual stepping capabilities of the DLRBiped robot [22], we define $R$ based on a shape $\mathcal{S}$ made of two portions of disks moving together in translation and rotation in the 2D plane (see Fig. 5). $\mathcal{S}$ depends on its specific position and orientation, i.e. an element $(x, y, \theta)$ of $S E(2)$. $R$ allows steps from the stance $\left(e_{l}, e_{r}\right)$ to the stance $\left(e_{l}^{\prime}, e_{r}^{\prime}\right)$ if and only if:

1) there exists a position and orientation $(x, y, \theta)$ of $\mathcal{S}$ such that the positions of $e_{l}$ and $e_{l}^{\prime}$ are inside the left part of $\mathcal{S}$, and the positions of $e_{r}$ and $e_{r}^{\prime}$ inside its right part;

2) with the same $\theta$, the orientations of $e_{l}$ and $e_{l}^{\prime}$ are in $\left[\theta-\frac{\alpha_{\text {in }}}{2}, \theta+\frac{\alpha_{\text {out }}}{2}\right]$ and the ones of $e_{r}$ and $e_{r}^{\prime}$ in $\left[\theta-\frac{\alpha_{o u t}}{2}, \theta+\frac{\alpha_{i n}}{2}\right], \alpha_{i n}$ and $\alpha_{\text {out }}$ being two empirically defined constants.

We pose $\Omega=S E(2)$. For $\chi=(x, y, \theta) \in \Omega$, we define $\mathcal{U}_{\chi}$ as the set of stances such that the center of the left foot is inside the left part of the shape $\mathcal{S}$ of position and orientation $\chi$, and the center of the right foot inside the right part.

To be computationally efficient, we use the following slightly conservative definition of weak collision-freeness: $\mathcal{U}_{\chi \in \Omega}$ is weakly collision-free if the shape $\mathcal{S}$ of configuration $\chi$ contains in its left and right parts the center of an entirely collision-free disk where the robot can safely put a foot.

With this new notion of weak collision-freeness, it can 


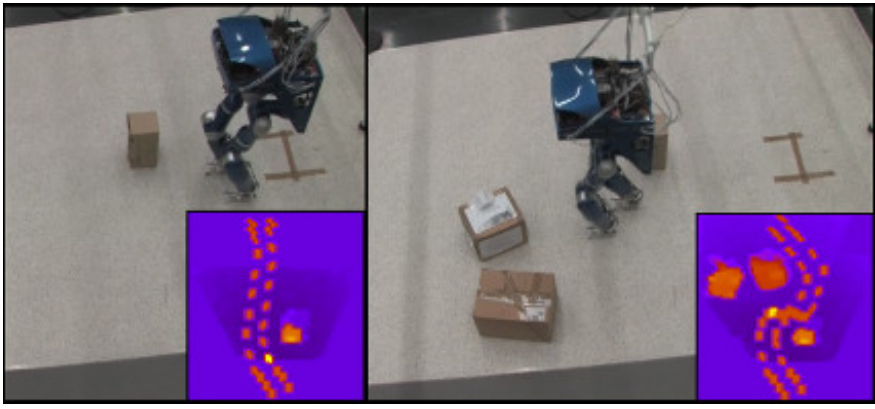

Fig. 7. An experiment of vision-based footstep planning with the DLR-Biped (boxes are thrown in front of it while it walks, and the robot is able to reactively replan a path to the goal).

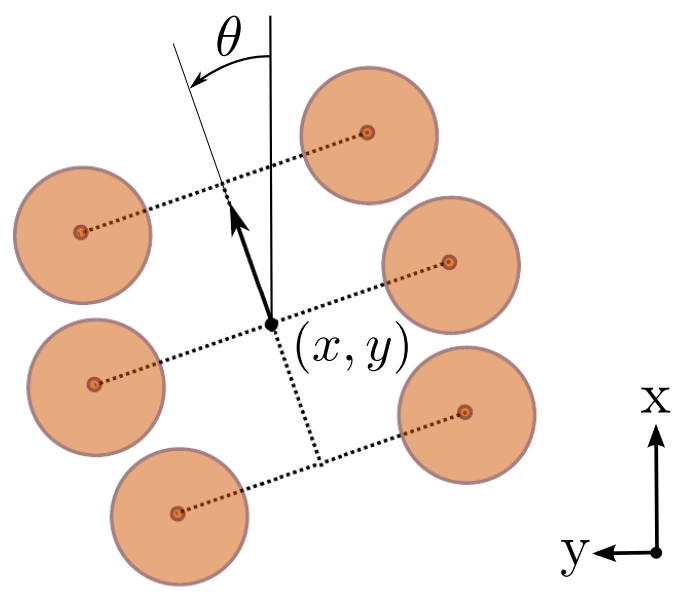

Fig. 8. The "2D shape" of six disks on which is based the definition of the transition relation $R$ that restricts the allowed configurations and steps for the hexapod (for each leg, the contact with the ground must be within the corresponding disk).

be shown that, as illustrated in Fig. 6, continuous weakly collision-free paths can easily (and greedily) be converted into valid sequences of steps.

The details necessary for an efficient implementation that deals with limited ground height variations can be found in [24]. Using a walking controller based on the concept of Divergent Component of Motion [9], heightmaps frequently updated through stereo vision, and the open source library OMPL [28] with RRT-Connect [18] to plan the continuous motion of the shape $\mathcal{S}$, we were able to realize online reactive vision-based footstep planning with the DLR-Biped robot (see Fig. 7). In [26], an additional parameter used to continuously modify the shape $S$ resulted in a slightly slower footstep planning algorithm for the robot HRP-2, but with extended stepping capabilities.

\section{APPLICATION TO LEGGED LOCOMOTION PLANNING FOR A HEXAPOD ROBOT}

In this section, we show that our method can also be used to plan the walking motion of a hexapod robot. Our objective is to make the hexapod walk on uneven terrain with non-gaited locomotion planning (which is typically computationally costly). The uneven terrain is described by a heightmap that sets

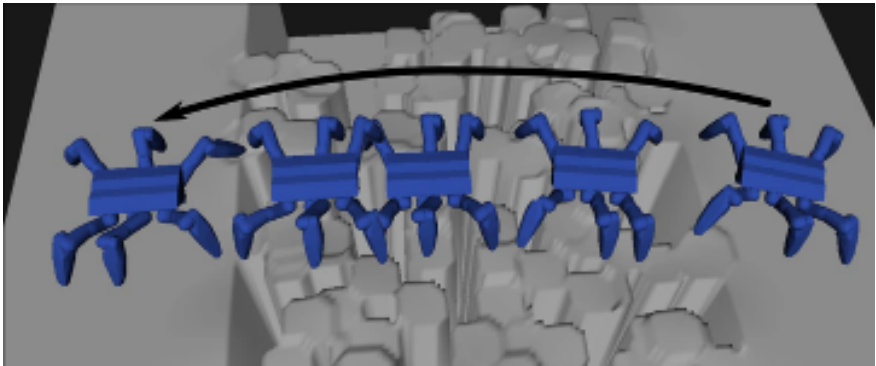

Fig. 9. The motion accross this terrain was planned in $57 \mathrm{~ms}$.

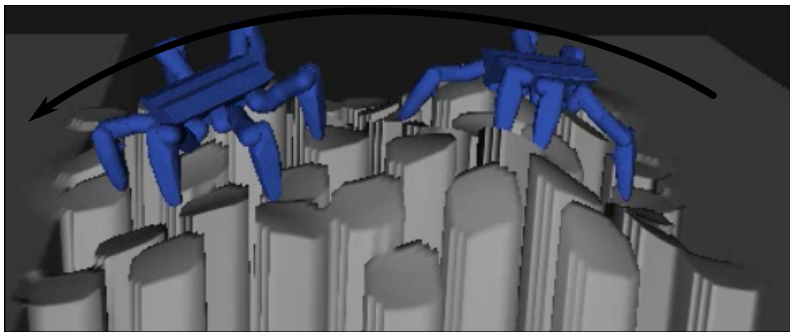

Fig. 10. The motion accross this more complex terrain was planned in $330 \mathrm{~ms}$.

the height of the contact positions. We ignore the contact orientations, so the configuration space of the hexapod is $\left(\mathbb{R}^{2}\right)^{6}$ (it is easy to define a heuristic that sets a unique whole-body configuration from the 6 contact positions; in particular, we require the robot main body to remain horizontal).

We define a new 2D shape adapted to the hexapod (see Fig. 8). It is a disjoint union of 6 open disks (one per leg). Again, we pose $\Omega=S E(2)$, and define that a given position and orientation of this shape is weakly collision-free if and only if each disk contains a location where the hexapod can safely put a foot (with a few additional restrictions on the differences of height between the footholds and on the maximum elevation of the heightmap in a neighboring area). Similarly to the definition of $R$ based on $\mathcal{S}$ in the previous section, we allow steps from a stance to another if there exists a configuration of the 2D shape in which both stances fit.

And again, we can prove that a greedy algorithm can efficiently transform any continuous weakly collision-free path into a valid sequence of steps for the hexapod, as shown in Fig. 1. As with the biped robot, our implementation uses OMPL and RRT-Connect for the continuous motion planning. We use a simple algorithm to detect steps that can be performed simultaneously (up to three legs of the hexapod can be moved at the same time).

This original technique for legged locomotion planning is convenient and fast: in the example described in Fig. 9 where the hexapod must go across an uneven and challenging terrain, the whole planning (continuous planning and twostage conversion into a discrete sequence of steps) was done in $57 \mathrm{~ms}$ on an Intel(R) Core(TM) i7 $1.60 \mathrm{GHz}$ CPU. The algorithm can even be improved to slightly change the roll and pitch of the hexapod according to the current height of its legs, which enables it to go across more complex terrains. For example, the motion accross the terrain shown in Fig. 10 was 
planned in $330 \mathrm{~ms}$. We cannot directly use the same method to solve planning problems as complex as the ones considered in [14], but it is an interesting compromise between gaited methods and more computationally costly ones such as [14].

\section{CONCLUSION}

We presented in this paper a method to solve different footstep planning problems for legged robots via continuous motion planning, using the notion of weak-collision freeness.

A precise comparison of algorithmic efficiency with respect to state-of-the-art approaches is beyond the scope of this paper, but we have successfully applied our general technique to various systems with computation times that allowed reactive motion planning. More importantly, our notion of weakcollision freeness involves particular sets of configurations that somehow abstract the footsteps and allow the planning of discrete actions to be casted as a completely continuous motion planning problem. This is conceptually different from state-ofthe-art approaches, and it provides a new way to reason about planning discrete actions in continuous spaces. We believe that it may have other applications than footstep or multi-contact planning, for example in dexterous manipulation or hybrid systems.

\section{REFERENCES}

[1] Boissonnat, J.D., Devillers, O., Lazard, S.: Motion planning of legged robots. SIAM Journal on Computing 30(1), 218-246 (2000)

[2] Bourgeot, J.M., Cislo, N., Espiau, B.: Path-planning and tracking in a 3D complex environment for an anthropomorphic biped robot. In: IEEE Int. Conf. on Intelligent Robots and Systems (IROS'02), vol. 3, pp. 2509-2514 (2002)

[3] Bouyarmane, K., Kheddar, A.: Multi-contact stances planning for multiple agents. In: IEEE Int. Conf. on Robotics and Automation (ICRA'11), pp. 5246 - 5253 (2011)

[4] Bullo, F., Lewis, A.D.: Geometric Control of Mechanical Systems, Texts in Applied Mathematics, vol. 49. Springer Verlag (2004)

[5] Chestnutt, J., Kuffner, J.J., Nishiwaki, K., Kagami, S.: Planning biped navigation strategies in complex environments. In: IEEE/RAS Int. Conf. on Humanoid Robots (Humanoids'03) (2003)

[6] Chestnutt, J., Lau, M., Cheung, G., Kuffner, J.J., Hodgins, J., Kanade, T.: Footstep planning for the honda asimo humanoid. In: IEEE Int. Conf. on Robotics and Automation (ICRA'05), pp. 631-636 (2005)

[7] Chestnutt, J., Nishiwaki, K., Kuffner, J.J., Kagami, S.: An adaptive action model for legged navigation planning. In: IEEE/RAS Int. Conf. on Humanoid Robots (Humanoids'07), pp. 196-202 (2007)

[8] Dalibard, S., El Khoury, A., Lamiraux, F., Taix, M., Laumond, J.P.: Small-space controllability of a walking humanoid robot. In: IEEE/RAS Int. Conf. on Humanoid Robots (Humanoids'11) (2011)

[9] Englsberger, J., Ott, C., Albu-Schaffer, A.: Three-dimensional bipedal walking control based on divergent component of motion. Robotics, IEEE Transactions on 31(2), 355-368 (2015)

[10] Goodwine, B., Burdick, J.W.: Controllability of kinematic control systems on stratified configuration spaces. IEEE Transactions on Automatic Control 46(3), 358-368 (2001)

[11] Goodwine, B., Burdick, J.W.: Motion planning for kinematic stratified systems with application to quasi-static legged locomotion and finger gaiting. IEEE Transactions on Robotics and Automation 18(2), 209$222(2001)$

[12] Gutmann, J.S., Fukuchi, M., Fujita, M.: Real-time path planning for humanoid robot navigation. In: Int. Joint Conf. on Artificial Intelligence (IJCAI05), pp. 1232-1237 (2005)

[13] Harmati, I., Kiss, B.: Motion planning algorithms for stratified kinematic systems with application to hexapod robot. Journal of Acta Cybernetica 15(2), 225-240 (2001)

[14] Hauser, K., Bretl, T., Latombe, J.C., Wilcox, B.: Motion planning for a six-legged lunar robot. In: 7th Workshop on the Algorithmic Foundations of Robotics (WAFR'06), pp. 16-18 (2006)
[15] Hornung, A., Dornbush, A., Likhachev, M., Bennewitz, M.: Anytime search-based footstep planning with suboptimality bounds. In: IEEE/RAS Int. Conf. on Humanoid Robots (Humanoids'12), pp. 674679 (2012)

[16] Kanoun, O., Laumond, J.P., Yoshida, E.: Planning foot placements for a humanoid robot: A problem of inverse kinematics. Int. Journal of Robotics Research 30(4), 476-485 (2011)

[17] Kavraki, L.E., Svestka, P., Latombe, J.C., Overmars, M.H.: Probabilistic roadmaps for path planning in high-dimensional configuration spaces. IEEE Trans. on Robotics and Automation 12, 566-580 (1996)

[18] Kuffner, J.J., Lavalle, S.: RRT-Connect: An efficient approach to singlequery path planning. In: IEEE Int. Conf. on Robotics and Automation (ICRA'00), pp. 995-1001 (2000)

[19] Kuffner, J.J., Nishiwaki, K., Kagami, S., Inaba, M., Inoue, H.: Footstep planning among obstacles for biped robots. In: IEEE/RSJ Int. Conf. on Intelligent Robots and Systems (IROS'01), pp. 500-505 (2001)

[20] Lafferriere, G., Sussmann, H.J.: Motion planning for controllable systems without drift. In: IEEE Int. Conf. on Robotics and Automation (ICRA'91), pp. 1148-1153 (1991)

[21] Lavalle, S.M., Kuffner, J.J., Jr.: Rapidly-exploring random trees: Progress and prospects. In: Algorithmic and Computational Robotics: New Directions, pp. 293-308 (2000)

[22] Ott, C., Baumgartner, C., Mayr, J., Fuchs, M., Burger, R., Dongheui, L., Eiberger, O., Albu-Schäffer, A., Grebenstein, M., Hirzinger, G.: Development of a biped robot with torque controlled joints. In: IEEE/RAS Int. Conf. on Humanoid Robots (Humanoids'10), pp. 167173 (2010)

[23] Perrin, N.: From discrete to continuous motion planning. 10th Workshop on the Algorithmic Foundations of Robotics (WAFR'12) (2012)

[24] Perrin, N., Ott, C., Englsberger, J., Stasse, O., Lamiraux, F., Caldwell Darwin, G.: A Continuous Approach to Legged Locomotion Planning (2013). URL https://hal.archives-ouvertes.fr/hal-00805981

[25] Perrin, N., Stasse, O., Lamiraux, F., Kim, Y.J., Manocha, D.: Realtime footstep planning for humanoid robots among 3D obstacles using a hybrid bounding box. In: IEEE Int. Conf. on Robotics and Automation (ICRA'12) (2012)

[26] Perrin, N., Stasse, O., Lamiraux, F., Yoshida, E.: Weakly collision-free paths for continuous humanoid footstep planning. In: IEEE/RSJ Int. Conf. on Intelligent Robots and Systems (IROS'11), pp. 4408-4413 (2011)

[27] Sastry, S.: Nonlinear Systems: Analysis, Stability, and Control, Interdisciplinary Applied Mathematics, vol. 10. Springer (1999)

[28] Şucan, I.A., Moll, M., Kavraki, L.E.: The Open Motion Planning Library. IEEE Robotics \& Automation Magazine 19(4), 72-82 (2012). http://ompl.kavrakilab.org

[29] Yoshida, E., Esteves, C., Belousov, I., Laumond, J.P., Sakaguchi, T. Yokoi, K.: Planning 3D collision-free dynamic robotic motion through iterative reshaping. IEEE Trans. on Robotics 24(5), 1186-1198 (2008) 